\title{
Benign metastasizing leiomyoma of the lung: A case report and literature review
}

\author{
WEI-TIAN WEI and PENG-CHENG CHEN

\begin{abstract}
Department of Oncological Surgery, Zhejiang Cancer Hospital, Hangzhou, Zhejiang 310000, P.R. China
\end{abstract}
Received August 7, 2014; Accepted April 14, 2015

DOI: 10.3892/ol.2015.3224

\begin{abstract}
Pulmonary benign metastasizing leiomyoma (BML) is a rare event characterized by benign soft-tissue tumors that occur when uterine leiomyomas metastasize to the lung. The present study reports the case of a 47-year-old female patient who presented with multiple bilateral pulmonary nodules on a chest X-ray during a health checkup nine years after a hysterectomy due to uterine fibroids. Chest computed tomography $(\mathrm{CT})$ revealed multiple well-defined nodular shadows in the lung. One tumor of the left upper lung was resected by thoracoscopic surgery. Pathologically, the resected lesion consisted of benign spindle cells and was diagnosed as BML. The post-operative course was uneventful. Other lung nodules have been meticulously monitored at follow-up, and repeat CT two years later showed that these nodules had not increased at all in size and that no new lobe nodules had appeared. The present study indicates that pulmonary BML occurs in a low proportion of female with a history of uterine leiomyoma and treatment methods for it are diverse and controversial.
\end{abstract}

\section{Introduction}

The main feature of malignant disease is metastasis, the ability of cancer cells to spread from a primary site to form tumors at distant sites in the body. However, even when certain non-cancerous tumors appear to be benign, a number exhibit low-grade clinically malignant behavior and present as rare manifestations. For example, uterine leiomyoma, the most common type of uterine tumor, is known as a benign tumor. However, an unusual growth pattern is evident at presentation in a small proportion of uterine leiomyomas, which are subsequently termed benign metastasizing leiomyomas (BMLs). BML is a poorly-defined clinicopathological condition that features histologically benign metastatic smooth muscle tumors (1) characterized by the proliferation of, usually multiple, smooth muscle nodules. BML is a rare cause of

Correspondence to: Dr Peng-Cheng Chen, Department of Oncological Surgery, Zhejiang Cancer Hospital, 38 Guangji Road, Hangzhou, Zhejiang 310000, P.R. China

E-mail: chenpc0425@126.com

Key words: benign metastasizing leiomyoma, pulmonary nodules pulmonary nodules that occur upon the metastasis of uterine leiomyomas to the lung (2). As the majority of tumors are asymptomatic, they are therefore identified incidentally on routine chest X-rays, however, certain of these tumors induce coughing, hemoptysis, dyspnea and decreased pulmonary function $(3,4)$. Although $\sim 100$ cases have been reported in the literature (5), the incidence, pathogenesis and treatment remain ambiguous. Due to the low morbidity and scarcity of reports on this condition, there is no consensus on which methods should be used to treat this disease. The current study reports a case of pulmonary BML, with a history of hysterectomy due to uterine leiomyoma, and presents a brief review with regard to the diagnosis and treatment of this disease.

\section{Case report}

A 47-year-old female presented with multiple bilateral lung nodules discovered on pre-operative imaging. Upon admission, the patient was in a good general condition. The patient had undergone a hysterectomy 9 years previously for leiomyoma of the uterus, but had not previously suffered from pulmonary disease, such as pulmonary tuberculosis or pneumonia. No chest symptoms, including coughing, dyspnea, chest pain or tightness, were present. Chest tomography (CT) identified multiple well-defined nodular shadows in the lungs (Fig. 1). These lesions showed no avidity upon positron emission tomography (Fig. 2). The patient was otherwise in good health. The blood chemistry data were unremarkable, and the carcinoembryonic antigen, neuron-specific enolase, squamous cell carcinoma-related antigen and carbohydrate antigen 125 tumor marker values were all within normal limits. BML, malignant metastatic tumors or epithelioid hemangioendothelioma were suspected when admitted to the Zhejiang Cancer Hospital (Hangzhou, Zhejiang, China). The patient subsequently underwent video-assisted wedge resection of the left upper lobe supported the suspected diagnosis. The post-operative recovery was uneventful. The lesions excised from the left upper lung lobe indicated low-grade spindle cell proliferation consistent with BML (Fig. 3). The post-operative pathological report revealed epithelial adenomatoid hyperplasia of the smooth muscle and the hyperplasia of the alveolar epithelium, which was initially considered to be leiomyomatous hamartoma and alveolar 


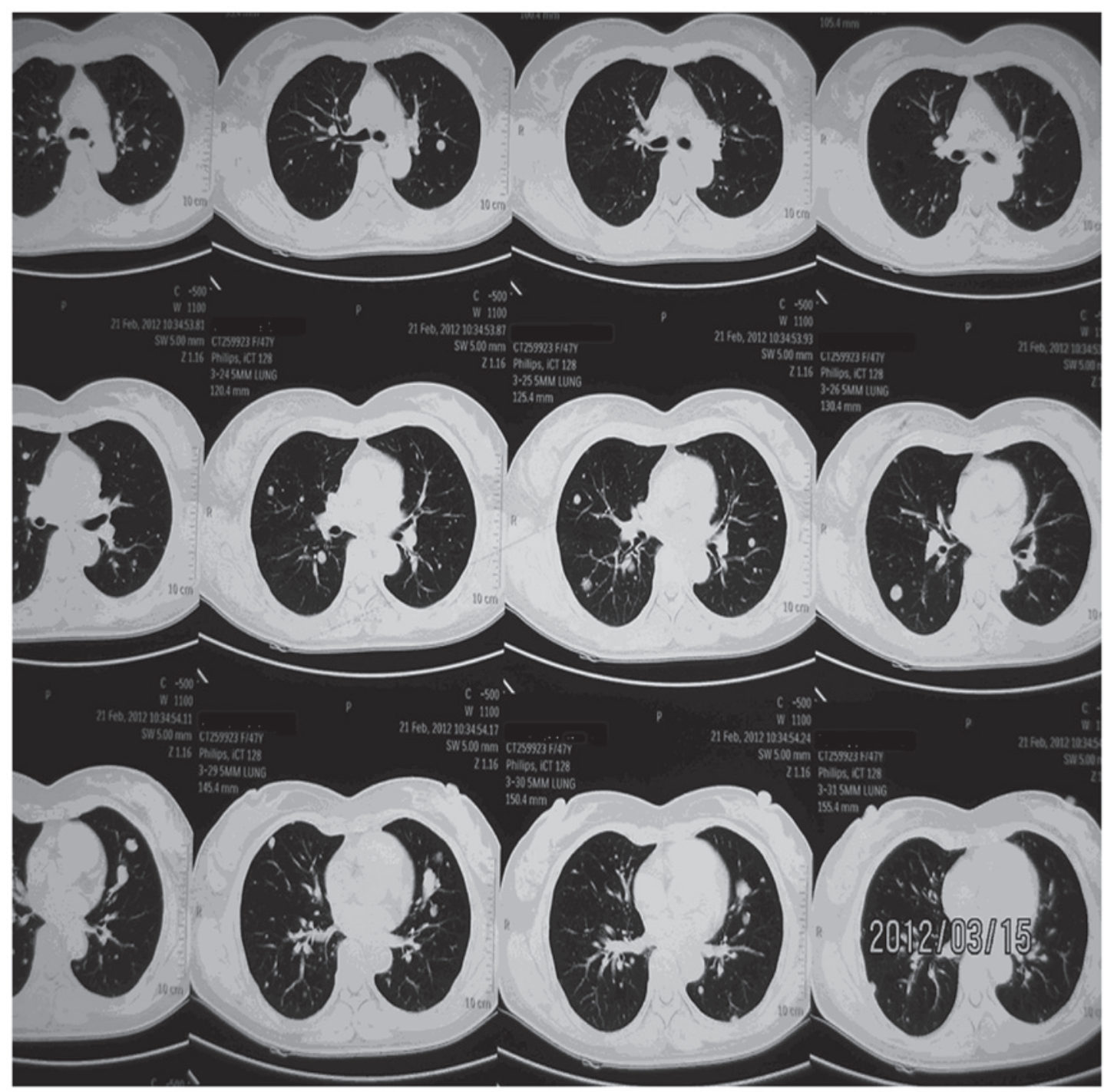

Figure 1. Computed tomography scans showing generally smooth and diffusely distributed lesions in each lung.

epithelial hyperplasia (multicentric, with a 2-cm diameter for the larger nodule and a $0.2-\mathrm{cm}$ diameter for the smaller nodule). The lesions were entirely mesenchymal with entrapped epithelial elements. Histopathological examination showed that the tumor consisted of well-differentiated spindle-shaped cells, with low nuclear and cellular variance in size and shape; there was no evident nuclear atypia and mitotic figures. Immunohistochemical studies revealed spindle cells positive for actin (Fig. 4A), desmin (Fig. 4B) and smooth muscle actin (Fig. 4C). The entrapped epithelium in the tissue was positive for cytokeratin 7 (Fig. 4D), surfactant protein A (Fig. 4E), and thyroid transcription factor 1 (Fig. 4F). The $\mathrm{Ki}-67$ positivity rate was low, at $\sim 5 \%$ of the spindle cells. This profile supported the light microscopic impression. In the pathological report, the pathologist indicated that the disease of this patient may be BML, and decided that a diagnosis could be formed by combining the pathological report with the clinical data.

The patient followed an uneventful post-operative course and was discharged on the third post-operative day. The patient is currently being followed up on an outpatient basis without any treatment. At present, more than two years after surgery, repeated CT (Fig. 5) scans showed that the number and size of the lung nodules remain unchanged.

\section{Discussion}

BML is a rare condition often occurring in middle-aged women with a history of uterine leiomyomata. Although the biological behavior of BML suggested malignancy, the tumor is borderline, with benign histological features. A previous stuy demonstrated that the lungs are the most common site of metastases (6). Pulmonary BML may present as asymptomatic pulmonary nodules diagnosed incidentally, be synchronous with uterine leiomyomas or arise following hysterectomy. Surgical palliation could be an option in highly selected patients, but the results are debatable. Thoracic surgeons should be aware of BML as a cause of multiple pulmonary nodules.

Uterine leiomyoma is the most frequently occurring form of uterine tumor and is typically considered to be benign, with a favorable long-term prognosis. BML has been described as incidental pulmonary nodules in women with a history of uterine leiomyomas (7). The pathogenesis of BML of the lung 


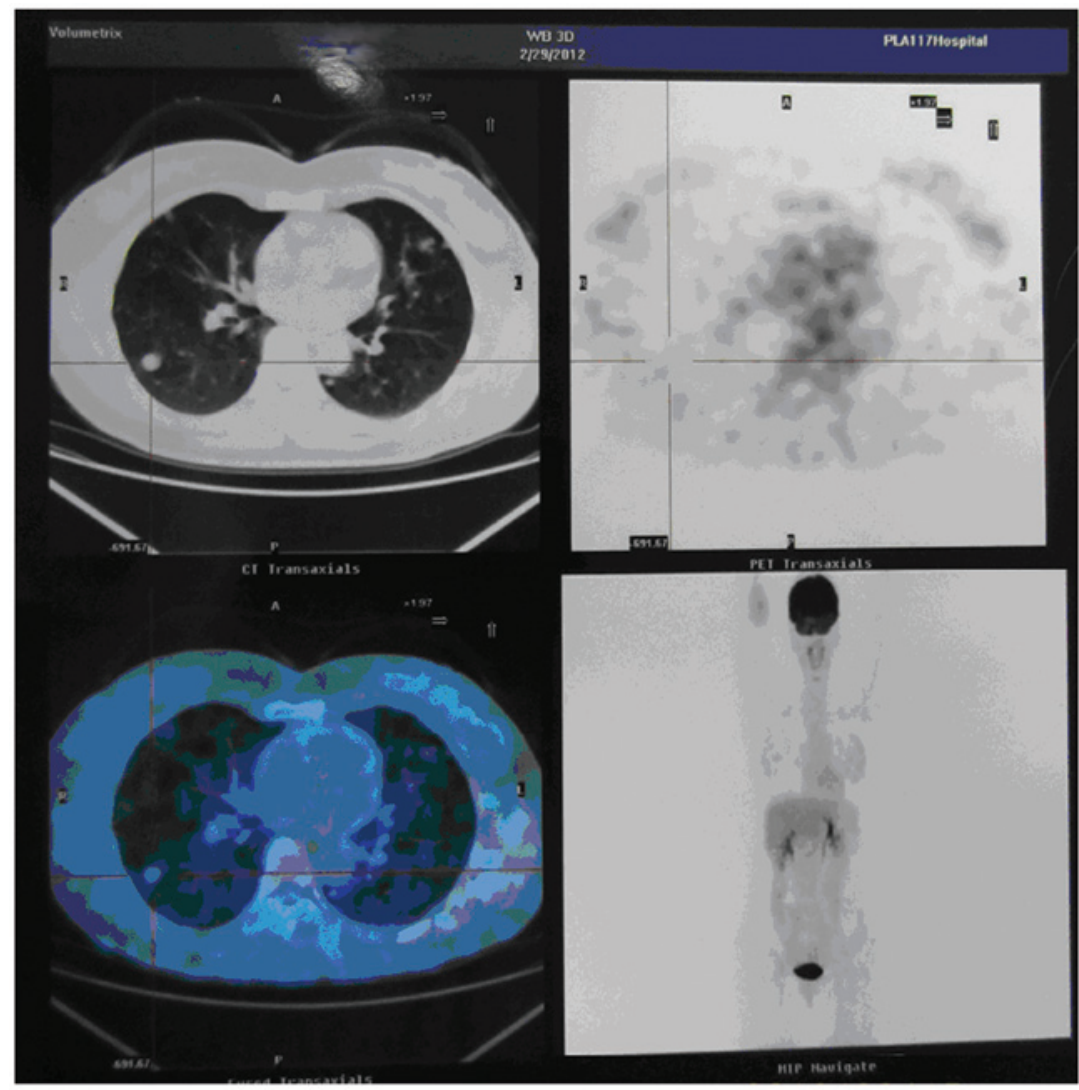

Figure 2. Positron emission tomography-computed tomography results revealing multiple hypometabolic masses in the lungs.

A

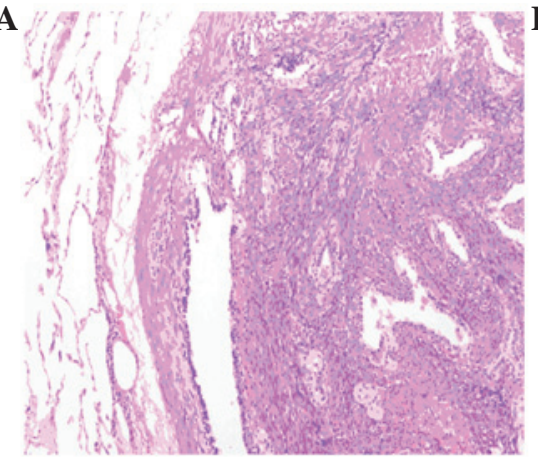

B

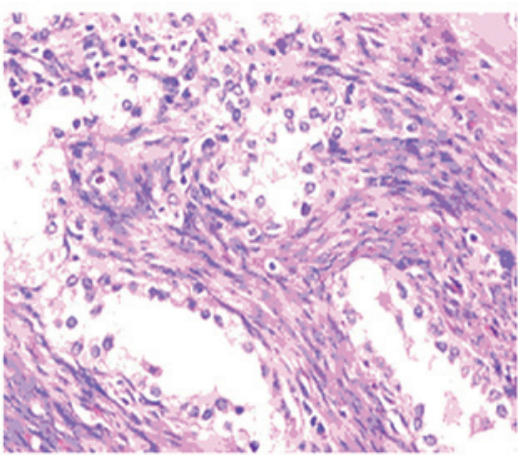

Figure 3. Low-grade spindle cell proliferation in the excised lesions from the left upper lung lobe. (A) Benign metastasizing leiomyoma (BML) in the lung with entrapped bronchoalveolar epithelium (hematoxylin and eosin staining, x10 magnification). (B) BML in the lung showing benign-appearing spindle-shaped cells arranged in intersecting fascicles (hematoxylin and eosin staining, x40 magnification).

has not yet been completely identified. The disease occurs predominantly in women of reproductive age (8), particularly during the premenopausal period. There is lack of clinicopathological details and follow-up data on these neoplasms, and the histological diagnosis is challenging and usually problematic. The first case of pulmonary BML was described in a study by Steiner (9) in 1939, which reported the case of a 36-year-old female who succumbed to cor pulmonale arising secondary to multiple pulmonary metastases from benign uterine leiomyomas. In 1983, Martin (10) classified leiomyomatous lesions of the lung into three large groups: Leiomyomatosis in women, metastatic leiomyoma in men/children, and multiple pulmonary fibroleiomyomatous hamartomas occurring in any subjects. To date, the youngest reported patient with pulmonary BML has been 23 years old (3). Occasionally, the pulmonary lesions can be found at the same time as the uterine leiomyoma (11). The mean duration between hysterectomy and the appearance of lung lesions is $\sim 15$ years (12). The majority of reported cases occur in women of reproductive age following hysterectomy or surgery for uterine leiomyomas. The natural history of pulmonary BML remains uncertain (13). Although the disease has benign histological features and often presents with indolent features, its metastatic behavior suggests its malignant potential. It has been suggested that pulmonary BML represents a low-grade, slow-growing leiomyosarcoma $(14,15)$. Giove et al $(16)$ reported a case of BML in a 55-year-old female who remained alive with lung, lymph node, skin, bone and possible brain metastases 14 years after the 

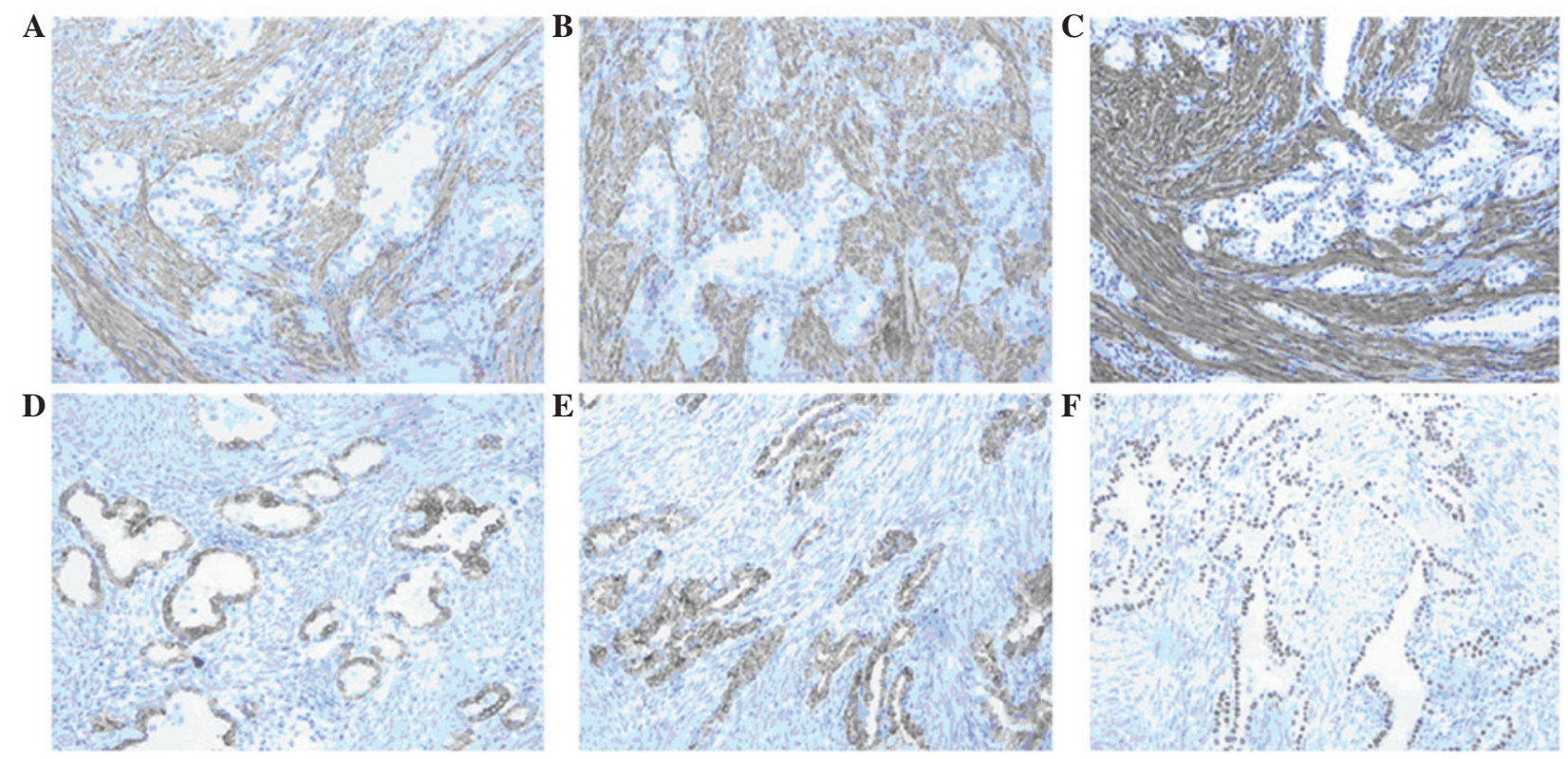

Figure 4. Immunohistochemical staining of benign metastasizing leiomyoma (BML). BML in the lung showing positivity for (A) actin, (B) desmin and (C) smooth muscle actin, with entrapped bronchoalveolar epithelium showing positivity for (D) cytokeratin 7, (E) surfactant protein A and (F) thyroid transcription factor 1.
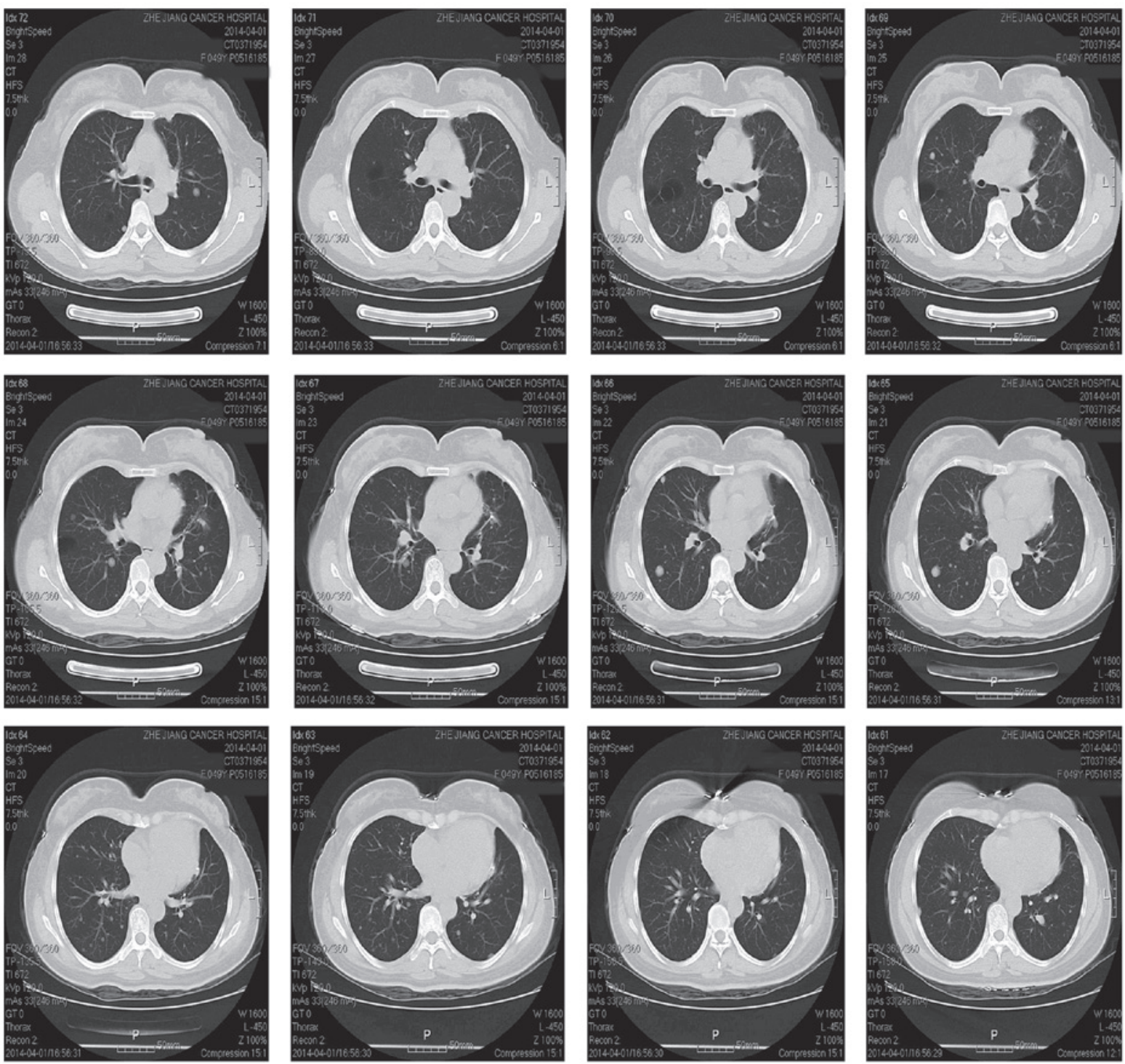

Figure 5. Repeated computed tomography scan at 2 years post-diagnosis. The existing nodules have remained stable and no new nodules can be observed. 
first uterine myomectomy. Jautzke et al (17) reviewed 74 cases of BML and found the lungs to be the most common site of involvement, similar to the study by Rivera et $a l$, which found pulmonary lesions in 20 out of 33 cases (8). Although the lungs are the most common sites for metastasis, extrapulmonary lesions have been documented in the lymph nodes, deep soft tissues, omentum and mesentery, bone, spine, skull base and heart (5). The majority of tumors are asymptomatic and are found incidentally on routine chest roentgenograms, however, coughing, dyspnea and chest pain have also been reported as symptoms (18). Recently, Miyazaki et al (19) presented a case of massive hemoptysis from pulmonary BML, and transarterial embolization was attempted in the treatment as the thoracic surgeon expected that resection of the tumor may be difficult due to the tumor location. The majority of reports of BML describe a chronic, benign, indolent course, but Bachman and Wolff reported a case of mortality due to of acute respiratory distress syndrome from multiple lesions with massive pulmonary and hilar lymphatic metastasis (20).

Pathological features of pulmonary BML are often of a benign nature, as observed in the present case. Absence of high cellularity coagulative tumor cell necrosis, cytological atypia and increased mitosis ( $>5$ per 10 high-powered fields) with a low Ki-67 index support the low proliferative state and benign nature of these tumors (5). Interlacing fascicles of smooth muscle cells lacking anaplasia or vascular invasion, with entrapped respiratory epithelium are revealed upon histological examination. A range of immunohistochemical markers, including desmin and muscle-specific actin, are present to confirm the mesenchymal derivation of these tumors with smooth muscle differentiation. In addition, the presence of estrogen and progesterone receptors supports the derivation of BML from the uterus $(21,22)$, which reinforces the use of treatment with hormonal agents. Radiographically, BML presents as solitary or multiple lesions scattered within the normal interstitium; these well-circumscribed nodules range from a few millimeters to a few centimeters in size. Intravenous contrast medium does not enhance the nodules. Endobronchial and pleural sparing is also characteristic of BML. Rare cases have been reported with a miliary pattern (23), cavitary lung nodules, interstitial lung disease and multiloculated fluid-containing cystic lesions (24). The present patient was finally diagnosed with pulmonary BML by combining the pathological report with the clinical data.

No standard management guidelines have been formulated with regard to the treatment of BML. Careful observation, surgical resection, hysterectomy and bilateral oophorectomy, administration of progestins and aromatase inhibitors, and medical castration using luteinizing hormone-releasing hormone analogs have all been reported as potential treatment modalities (25). Lesions that increase in size may require surgical resection to prevent potentially fatal complications such as massive hemoptysis. However, the effect of reducing the tumor burden through surgical palliation should be carefully evaluated. Smaller subcentimeter lesions can be followed with surveillance scans. The presence of estrogen and progesterone receptors makes these tumors susceptible to hormonal manipulation by surgical or medical castration. Hormonal relative treatment is a commonly chosen therapy for BML when estrogen and progesterone receptors are identified on tumor histology $(7,18)$. Certain patients have been shown to be sensitive to treatment with progestin, goserelin, ovarian ablation and oophorectomy. This suggests that estrogen and progesterone may play a significant role in the pathogenesis of BML. Bilateral oophorectomy or medical reversible castration with luteinizing hormone-releasing hormone analogs control gonadal hormone secretion $(1,26)$, which may control the growth of already established lesions. However, hormone therapy does not generate a response in all patients, and the side-effects of flushes, fatigue and nausea can be aggravating to the patient (3). In a previous study, even in the presence of positive estrogen and progesterone receptors on smooth muscle cells, no significant change was noted in the size of the BML lesions following 6-12 months of treatment with tamoxifen, progesterone and an aromatase inhibitor (7). A combination of medical and surgical treatment would exert a synergistic effect and should be considered in the management of progressive and symptomatic lesions. One previous study proposed that BML may naturally decrease following the menopause (27). The majority of BML lesions remain at one size, however, a small percentage display an aggressive course (28). It is currently unclear why the tumor progress in BML. As the majority of lesions stain positive for estrogen and progesterone receptors, and as the present patient refused hormone therapy, the estrogen and progesterone receptors were not detected in the histological chemistry examination. Therefore, a wait-and-see strategy was decided upon for the patient.

In the current study, the case of a female with pulmonary BML arising after hysterectomy, who was followed up after surgery, was reviewed. Although a rare disease, BML should be considered by physicians for asymptomatic women of reproductive age with a history of uterine leiomyoma, who present with solitary or multiple pulmonary nodules. A standard strategy remains to be established for the treatment of this disease, but as the clinical course of BML varies among cases, an individual treatment approach should be considered. Thoracic surgeons should be aware of this unusual cause of multiple pulmonary nodules.

\section{References}

1. Egberts JH, Schafmayer C, Bauerschlag DO, Jänig U and Tepel J: Benign abdominal and pulmonary metastasizing leiomyoma of the uterus. Arch Gynecol Obstet 274: 319-322, 2006.

2. Banner AS, Carrington CB, Emory WB, et al: Efficacy of oophorectomy in lymphangioleiomyomatosis and benign metastasizing leiomyoma. N Engl J Med 305: 204-209, 1981.

3. Goyle KK, Moore DF Jr, Garrett C and Goyle V: Benign metastasizing leiomyomatosis: Case report and review. Am J Clin Oncol 26: 473-476, 2003.

4. Säynäjäkängas $O$, Maiche AG and Liakka KA: Multiple progressive pulmonary leiomyomatous metastases treated with tamoxifen - a case report with a review of the literature. Acta Oncol 43: 113-114, 2004.

5. Rege AS, Snyder JA and Scott WJ: Benign metastasizing leiomyoma: A rare cause of multiple pulmonary nodules. Ann Thorac Surg 93: e149-e151, 2012.

6. Chen S, Zhang Y, Zhang J, et al: Pulmonary benign metastasizing leiomyoma from uterine leiomyoma. World J Surg Oncol 11: 163, 2013.

7. Abramson S, Gilkeson RC, Goldstein JD, Woodard PK, Eisenberg $\mathrm{R}$ and Abramson N: Benign metastasizing leiomyoma: Clinical, imaging and pathologic correlation. AJR Am J Roentgenol 176: 1409-1413, 2001. 
8. Rivera JA, Christopoulos S, Small D and Trifiro M: Hormonal manipulation of benign metastasizing leiomyomas: Report of two cases and review of the literature. J Clin Endocrinol Metab 89: 3183-3188, 2004

9. Steiner PE: Metastasizing fibroleiomyoma of the uterus: Report of a case and review of the literature. Am J Pathol 15: 89-110, 1939.

10. Martin E: Leiomyomatous lung lesions: A proposed classification. AJR Am J Roentgenol 141: 269-272, 1983.

11. Moon H, Park SJ, Lee HB, et al: Pulmonary benign metastasizing leiomyoma in a postmenopausal woman. Am J Med Sci 338 72-74, 2009.

12. Kayser K, Zink S, Schneider T, et al: Benign metastasizing leiomyoma of the uterus: Documentation of clinical, immunohistochemical and lectin-histochemical data of ten cases. Virchows Arch 437: 284-292, 2000.

13. Ng JS, Han A, Chew SH and Low J: A clinicopathologic study of uterine smooth muscle tumours of uncertain malignant potential (STUMP). Ann Acad Med Singapore 39: 625-628, 2010.

14. Burkhardt A, Otto HF and Kaukel E: Multiple pulmonary (hamartomatous?) leiomyomas. Light and electron microscopic study. Virchows Arch A Pathol Anat Histol 394: 133-141, 1981.

15. Cohen DT, Oliva E, Hahn PF, Fuller AF Jr and Lee SI: Uterine smooth-muscle tumors with unusual growth patterns: Imaging with pathologic correlation. AJR Am J Roentgenol 188: 246-255, 2007.

16. Giove S, Scappaticci E, Baldi S, Ricci C and Minetto E: Benign metastasizing leiomyoma of the uterus. Case report. Minerva Med 75: 1819-1821, 1984 (In Italian).

17. Jautzke G, Muller-Ruchholtz E and Thalmann U: Immunohistological detection of estrogen and progesterone receptors in multiple and well differentiated leiomyomatous lung tumors in women with uterine leiomyomas (so-called benign metastasizing leiomyomas). A report on 5 cases. Pathol Res Pract 192: 215-223, 1996.
18. Ogawa M, Hara M, Ozawa Y, et al: Benign metastasizing leiomyoma of the lung with malignant transformation mimicking mediastinal tumor. Clin Imaging 35: 401-404, 2011.

19. Miyazaki M, Nakayama A, Noda D, Maehara Y and Tsushima Y: Difficulty in complete transarterial embolization for pulmonary benign metastasizing leiomyoma with massive hemoptysis. Jpn J Radiol 32: 53-57, 2014.

20. Bachman D and Wolff M: Pulmonary metastases from benign-appearing smooth muscle tumors of the uterus. AJR Am J Roentgenol 127: 441-446, 1976.

21. Rao UN, Finkelstein SD and Jones MW: Comparative immunohistochemical and molecular analysis of uterine and extrauterine leiomyosarcomas. Mod Pathol 12: 1001-1009, 1999.

22. McGinley KM, Bryant S, Kattine AA, Fitzgibbon JF and Googe PB: Cutaneous leiomyomas lack estrogen and progesterone receptor immunoreactivity. J Cutan Pathol 24: 241-245, 1997.

23. Lipton JH, Fong TC and Burgess KR: Miliary pattern as presentation of leiomyomatosis of the lung. Chest 91: 781-782, 1987.

24. Osadchy A, Zehavi T and Zissin R: Pulmonary benign metastasising leiomyomas presenting as fluid-containing masses on $\mathrm{CT}$ in a patient with two unrelated malignancies. Br J Radiol 78: 639-641, 2005.

25. Yoon G, Kim TJ, Sung CO, et al: Benign metastasizing leiomyoma with multiple lymph node metastasis: A case report. Cancer Res Treat 43: 131-133, 2011.

26. Arif S, Ganesan R and Spooner D: Intravascular leiomyomatosis and benign metastasizing leiomyoma: An unusual case. Int J Gynecol Cancer 16: 1448-1450, 2006.

27. Arai T, Yasuda Y, Takaya T and Shibayama M: Natural decrease of benign metastasizing leiomyoma. Chest 117: 921-922, 2000.

28. Sapmaz F, Ergin M, Katrancioglu O, Gonlugur T, Gonlugur U and Elagoz S: Benign metastasizing leiomyoma. Lung 186: 271-273, 2008. 